\title{
Multi-peak bound states for nonlinear Schrödinger equations
}

\author{
by \\ Manuel DEL PINO ${ }^{1}$ \\ Departamento de Maltematicas, Fac. de Ciencias, \\ Universidad de Chile Casilla 653, Santiago 1, Chile \\ E-mail: delpino@dim.uchile.cl \\ and \\ Patricio L. FELMER ${ }^{2}$ \\ Departamento de Ingeniería Matemática F.C.F.M. Universidad de Chile, \\ Casilla 170 Correo 3, Santiago, Chile \\ E-mail: plelmer@dim.uchile.cl
}

ABSTRACT. - In this paper we consider the study of standing wave solutions for a nonlinear Schrödinger equation. This problem reduces to that of finding nonnegative solutions of

$$
\varepsilon^{2} \Delta u-V(x) u+f(u)=0 \text { in } \Omega
$$

with finite energy. Here $\varepsilon$ is a small parameter, $\Omega$ is a smooth, possibly unbounded domain, $f$ is an appropriate superlinear function, and $V$ is a positive potential, bounded away from zero.

It is the purpose of this article to obtain multi-peak solutions in the "multiple well case". We find solutions exhibiting concentration at any prescribed finite set of local minima, possibly degenerate, of the potential.

The proof relies on variational arguments, where a penalization-type method is developed for the identification of the desired solutions. (C) 1998 L'Association Publications de l'Institut Henri Poincaré. Published by Elsevier B.V. All rights reserved

I. Partially supported by FONDECYT Grant 1950-303 and Grant CI1:CT93-0323 CCL.

2. Partially supported by FONDECYT Grant 1940-505. 
RÉSUMÉ. - Dans cet article, on considère l'étude des solutions de ondes permanentes d'une équation de Schrödinger nonlinéaire. Ce problème se réduit à la recherche de solutions non négatives de

$$
\varepsilon^{2} \Delta u-V(x) u+f(u)=0 \text { dians } \Omega .
$$

avec une énergie finie. Ici $\varepsilon$ est un paramètre petit, $\Omega$ est un domaine lisse qui peut être non borné, $f$ est une fonction superlinéaire appropriée et $V$ est un potentiel positif borné hors de zéro.

L'objectif de cet article consiste à obtenir des solutions à pics multiples dans le cas de puits multiples. Nous trouvons des solutions qui montrent une concentration pour tout ensemble choisi fini de minima locaux du potentiel, qui peuvent être dégénérés.

La démonstration se base sur des arguments variationnels, où une méthode de pénalisation est développée pour identifier les solutions cherchées. (C) 1998 L'Association Publications de l'Institut Henri Poincaré. Published by Elsevier B.V. All rights reserved

\section{INTRODUCTION}

The nonlinear Schrödinger equation in $\mathbb{R}^{N}$

$$
i \hbar \frac{\partial \psi}{\partial t}=-\frac{\hbar^{2}}{2 m} \Delta \psi+W(x) \psi-\gamma|\psi|^{p-1} \psi
$$

has been object of extensive research in recent years. In this paper we consider the study of standing waves of equation (0.1), namely of special solutions of the form $\psi(x, t)=\exp (-i E t / \hbar) v(x)$, where $v(x)>0$. It is easily checked that a $\psi$ of this form satisfies equation (0.1) if and only if the function $v(x)$ solves the elliptic equation

$$
\frac{\hbar^{2}}{2 m} \Delta v-(W(x)-E) v+\gamma v^{p}=0 .
$$

The problem we will study in this paper is that of existence of positive solutions with finite energy to this equation when $W-E$ is strictly positive, away from zero, and the potential $W$ exhibits multiple wells, namely several, possibly degenerate local minima. $\hbar$ will be regarded as a small parameter. After absorption of the parameters by scaling, the problem under consideration may be rewritten as

$$
\varepsilon^{2} \Delta u-V(x) u+u^{p}=0
$$


where $V \geq \alpha>0$ in $\mathbb{R}^{N}, N \geq 1$. In [5], Floer and Weinstein consider the case $N=1$ and $p=3$. For a given nondegenerate critical point of the potential $V$, assumed globally bounded, they construct a positive solution $u_{\varepsilon}$ to $(0.3)$, provided that $\varepsilon$ is sufficiently small. This solution concentrates around the critical point as $\varepsilon \rightarrow 0$, in the sense that its shape is a sharp peak near that point, while it almost vanishes everywhere else.

Their method, based on an interesting Lyapunov-Schmidt finite dimensional reduction, was extended by $\mathrm{Oh}$ in [8], [9] to conclude a similar result in higher dimensions, provided that $1<p<\frac{N+2}{N-2}$.

Oh restricts himself to potentials with "mild oscillation" at infinity, namely belonging to a Kato class. In case that $V$ is bounded this restriction is not necessary as noticed by Wang in [14]. Wang also observes that if $V$ is nonconstant and nondecreasing in one direction, then equation (0.3) has no solutions which tend to zero at infinity.

The method in [5] and [8] seems to rely in essential way on the nondegeneracy of the critical points. In [11], Rabinowitz lifted partially this requirement introducing a global variational technique to find a solution with "minimal energy" for all small $\varepsilon$, when $1<p<\frac{N+2}{N-2}$ and

$$
\liminf _{|x| \rightarrow \infty} V(x)>\inf _{x \in R^{v}} V(x) .
$$

Rabinowitz's approach actually covers a broader class of nonlinearities and the smallness of $\varepsilon$ is not required in case that the limit in the left of $(0.4)$ is $+\infty$. In [14], Wang established that this least energy (mountain pass) solution indeed concentrates around a global minimum of $V$ in the special case of equation $(0.3)$, as $\varepsilon \rightarrow 0$.

In [3], the authors succeeded in proving the local analogue of Wang's result. It is shown that if for an open, bounded set one has

$$
\inf _{\Lambda} V<\inf _{\partial \Lambda} V
$$

then $(0.3)$ possesses solutions $u_{\varepsilon}$ with just one local maximum, which concentrates around a minimum of $V$ in $\Lambda$. This local minimum may exhibit arbitrary degeneracy.

Concerning solutions with multiple concentration points, in [10] Oh applies the approach developed in [5] and [8] to construct a family of solutions $u_{\varepsilon}$ with peaks concentrating around any prescribed finite set of nondegenerate critical points of $V$ when $N=1$, and indicates how to proceed in higher dimensions where $1<p<\frac{N+2}{N-2}$.

It is the purpose of this article to obtain multi-peak solutions of $(0.3)$ in the "multiple well case", exhibiting concentration at any prescribed set of Vol. $15, n^{\circ} 2-1998$. 
possibly degenerate local minima of the potential, namely on $K$ disjoint sets $\Lambda$ where (0.5) holds.

We will actually consider a more general semilinear elliptic problem in a smooth domain $\Omega$, possibly unbounded, of the form

$$
\left\{\begin{array}{c}
\varepsilon^{2} \Delta u-V(x) u+f(u)=0 \text { in } \Omega \\
u \in H_{0}^{1}(\Omega), \quad u>0 \text { in } \Omega .
\end{array}\right.
$$

The potential $V$ will be assumed throughout this paper locally Hölder continuous and bounded below away from zero, say

$$
V(x) \geq \alpha>0 . \text { for all } x \in \Omega \text {. }
$$

We will also assume that $f: \mathbb{R}^{+} \rightarrow \mathbb{R}$ is of class $C^{1}$ and satisfies the following conditions.

(f1) $f(\xi)=o(\xi)$ near $\xi \geq 0$.

(f2) $\lim _{\xi \rightarrow \infty} \frac{f(\xi)}{\xi}=0$ for some $1<s<\frac{N+2}{N-2}$.

(f3) For some $2<\theta \leq s+1$ we have

$$
0 \leq \theta F(\xi)<f(\xi) \xi \text { for all } \xi>0
$$

where $F(\xi)=\int_{0}^{\xi} f(\tau) d \tau$.

(f4) The limiting equation

$$
\Delta u-b u+f(u)=0 \quad \text { in } \quad \mathbb{R}^{N}
$$

possesses a unique solution, up to translation, for any given $b>0$. Our main result for equation (0.6) is the following.

THEOREM 0.1. - Assume that there are bounded domains $\Lambda_{i}$, mutually disjoint, compactly contained in $\Omega, i=1, \ldots, K$, such that

$$
\inf _{\Lambda_{i}} V<\inf _{\partial \Lambda_{i}} V
$$

Then there is an $\varepsilon_{0}>0$ such that for every $0<\varepsilon<\varepsilon_{0}$ a positive solution $u_{\varepsilon} \in H_{0}^{1}(\Omega)$ to problem $(0.6)$ exists. Moreover, $u_{\varepsilon}$ possesses exactly $K$ local maxima $x_{\varepsilon, i}$, with $x_{\varepsilon, i}$ in $\Lambda_{i}$. We also have that $V\left(x_{\varepsilon, i}\right) \rightarrow \inf _{\Lambda_{i}} V$, and

$$
u_{\varepsilon}(x) \leq \alpha \exp \left(-\frac{\beta}{\varepsilon}\left|x-x_{\varepsilon, i}\right|\right)
$$

for all $x \in \Omega \backslash \cup_{j \neq i} \Lambda_{j}$, where $\alpha$ and $\beta$ are certain positive constants. 
We observe that no restriction on the global behavior of $V$ is required other than (0.7). In particular, $V$ is not required to be bounded or to belong to a Kato class.

On the other hand, the hypotheses on $f$ are milder than those required in [10]. In particular, hypothesis (f4) is satisfied by a large class of nonlinearities $f$ including $u^{p}, p>1$. See the work in Kwong and Zhang [6], and Chen and Lin [1]. Moreover, this assumption can be further relaxed to

(f4') The mountain pass value in $H_{0}^{1}(\Omega)$ of the energy functional associated to $(0.9)$ is the smallest nontrivial critical value and it is isolated.

See the remark at the end of $\S 2$.

It is interesting to observe the relationship between the result of Theorem 0.1 and the work by Coti-Zelati and Rabinowitz [2] on multibump solutions in spatially-periodic problems. For the case of equation (0.3), $1<p<\frac{N+2}{N-2}$, and $V$ periodic on each of its variables, it follows from their results that for a fixed, not necessarily small $\varepsilon$, solutions in $H^{1}\left(\mathbb{R}^{N}\right)$ with exactly $K$ bumps exist for each integer $K$, provided that the associated energy functional satisfies the so-called $(*)$ nondegeneracy condition. This assumption states that all critical points at energy levels in $[c, c+\delta)$ are isolated, where $c$ denotes the mountain pass minimax value of the associated functional, and $\delta>0$. These solutions have energy level close to $K c$. On the other hand, Theorem 0.1 is applicable to construct a $K$-bump solution in this situation when $\varepsilon$ is small, just assuming that $V$ possesses one local minimum. We do not know whether $(*)$ holds in this case.

The proof of Theorem 0.1 is variational, and uses ideas in the spirit of those in our previous work [3], where a penalization method enabling the identification of local mountain passes was developed. Roughly speaking, the main argument there consists of defining a suitable modification of the nonlinearity for which the mountain pass theorem is directly applicable to the associated energy. Then, taking advantage of the energy-minimality of the mountain pass solution, one finally shows that it becomes a solution to the original problem with the desired characteristics when $\varepsilon$ is sufficiently small.

Our current framework is more delicate, since the solutions we look for are at higher energy levels. They are not just rough mountain passes, so that energy-minimality is lost. We are able to overcome this difficulty by adding a new penalization term. In fact, we introduce a modification of the nonlinearity similar to that in [3] in order to prevent the occurrence of concentration outside the open sets $\Lambda_{i}$, and then a new term which provides a "balance" among the energies inside the distinct $\Lambda_{i}$ 's, in order to obtain exactly $K$-bumps. The solution is captured as a simple minimax quantity 
on a class of $K$-dimensional maps, and eventually shown to be a solution to the original equation with the appropriate features.

Finally, we would like to mention that the construction of solutions of (0.3) with an infinite number of bumps (hence not with finite energy) has been recently carried out by Thandi [13] in the nondegenerate case. Infinite-bump solutions in the framework of [2] were found by Spradlin in [12].

The organization of this paper is as follows: In 81 we define the modification of the functional needed for the proof of Theorem 0.1 , and prove some preliminary results. 32 is devoted to the proof of Theorem 0.1 .

\section{PRELIMINARIES}

This section is devoted to the definition and preliminary study of the penalized functional.

We introduce an appropriate penalization so that the concentration outside the sets $\Lambda_{i}$ is avoided and an adequate balance in the concentration is achieved. Then we prove that the penalized functional satisfies the Palais Smale condition (P.S.), and we set up the minimax scheme in order to obtain critical points of it. We provide next some estimates on the critical points.

In the framework of Theorem 0.1, associated to equation (0.6) we have the "energy" functional

$$
I_{\xi}(u)=\frac{1}{2} \int_{\Omega} \varepsilon^{2}|\nabla u|^{2}+V(x) u^{2}-\int_{\Omega} F(u),
$$

which is well defined for $u \in H$, where

$$
H=\left\{u \in H_{0}^{1}(\Omega) / \int_{\Omega 2} V(x) u^{2}<\infty\right\} .
$$

$H$ becomes a Hilbert space, continuously embedded in $H_{0}^{1}(\Omega)$, when endowed with the inner product

$$
<u, v>_{H}=\int_{\Omega} \nabla u \cdot \nabla v+V(x) u v
$$

whose associated norm we denote by $\|\cdot\|_{H}$.

In the definitions above, and in what follows we assume that $f, V$ and $\Omega$ satisfy the hypotheses of Theorem 0.1 , and that the function $f$ is extended 
as 0 on the negative axis. Under those assumptions it is standard to check that the nontrivial critical points of $I_{\varepsilon}$ correspond exactly to the positive classical solutions in $H_{0}^{1}(\Omega)$ of equation (0.6).

Next we define the first modification of our functional. Let $\alpha$ be as in $(0.7)$, and let us choose $a>0$ so that

$$
\frac{f(\xi)}{\xi} \leq \frac{\alpha}{2} \text { for all } 0 \leq \xi \leq a, \text { and } \frac{f(\xi)}{\xi} \geq \frac{f(a)}{a} \text { for all } \xi \geq a
$$

This choice of $a$ can be made since (f1) and (f3) hold. Let us set

$$
\tilde{f}(\xi)= \begin{cases}f(\xi) & \text { if } \quad \xi \leq a \\ \frac{f(a)}{a} \xi & \text { if } \quad \xi>a\end{cases}
$$

and define

$$
g(\cdot, \xi)=\chi_{\Lambda} f(\xi)+\left(1-\chi_{\Lambda}\right) \tilde{f}(\xi)
$$

where $\Lambda=\cup_{i=1}^{K^{i}} \Lambda_{i}$, with the bounded domains $\Lambda_{i}$ as in the assumptions of Theorem 0.1 , and $\chi_{\Lambda}$ denoting its characteristic function. It is easy to check that (f1)-(f3) implies that $g$ defined in this way is a Caratheodory function and it satisfies the following

(gl) $g(x, \xi)=o(\xi)$ near $\xi=0$ uniformly in $x \in \Omega$.

(g2) $\lim _{\xi \rightarrow \infty} \frac{g(x, \xi)}{\xi^{s}}=0$ uniformly in $x \in \Omega$, for some $1<s<\frac{N+2}{N-2}$ if $N \geq 3$, and no restriction on $s$ if $N=1.2$.

(g3) (i) $0<\theta G(x, \xi) \leq g(x, \xi) \xi \quad$ for all $x \in \Lambda, \xi>0$.

and

(ii) $0 \leq 2 G(x, \xi) \leq g(x, \xi) \xi \leq \frac{\alpha}{2} \xi^{2} \quad$ for all $\quad x \in \Omega \backslash \Lambda, \xi \in \mathbb{R}^{+}$.

Hcre we have denoted $G(x, \zeta)=\int_{0}^{\xi} g(x, \tau) d \tau$.

Now we define the modified functional $J_{\varepsilon}: H \rightarrow \mathbb{R}$ as

$$
J_{\varepsilon}(u)=\frac{1}{2} \int_{\Omega} \varepsilon^{2}|\nabla u|^{2}+V(x) u^{2}-\int_{\Omega} G(x, u) \quad, \quad u \in H .
$$

The functional $J_{\varepsilon}$ is of class $C^{1}$ in $H$ and its critical points are the positive solutions of the equation

$$
\varepsilon^{2} \Delta u-V(x) u+g(x, u)=0 \quad \text { in } \Omega .
$$

Next we introduce the second modification of the functional. For this purpose we assume, without loss of generality, that the sets $\Lambda_{i}$ have smooth 
boundary. We define the numbers $b_{i}=\operatorname{iuf}\left\{V(x) / x \in \Lambda_{i}\right\}$ and we let $\delta>0$ be so that

$$
\sup _{\Lambda} V \leq b_{i}+\delta
$$

We will necd the 'limiting functional' $I^{b}: H^{\perp}\left(\mathbb{R}^{N}\right) \rightarrow \mathbb{R}$ defincd as

$$
I^{b}(u)=\frac{1}{2} \int_{\mathbb{R}^{N}}|\nabla u|^{2}+b u^{2}-\int_{\mathbb{R}^{N}} F(u) .
$$

whose mountain pass critical value will be denoted by $c(b)$. Critical points of $I^{b}$ are the solutions of $(0.9)$. We define $c_{i}=c\left(b_{i}\right)$ and $\sigma_{i}=c\left(b_{i}+\delta\right)-c_{i}$. We assume that

$$
\sum_{i=1}^{K} \sigma_{i}<\frac{1}{2} \min \left\{c_{i} \mid i=1, \ldots K\right\}
$$

This can be achieved by making $\Lambda_{i}$ and $\delta$ smaller if necessary. It will be convenient to consider mutually disjoint open sets $\tilde{\Lambda}_{i}$ compactly containing $\Lambda_{i}$, for all $i=1, \ldots, K$. Then we define on $H$ the functional

$$
J_{\varepsilon}^{i}(u)=\frac{1}{2} \int_{\tilde{\Lambda}_{i}} \varepsilon^{2}|\nabla u|^{2}+V(x) u^{2}-\int_{\tilde{\Lambda}_{i}} G(x, u), \quad u \in H .
$$

and the penalization

$$
P_{\varepsilon}(u)=M \sum_{i=1}^{K}\left\{\left(J_{\varepsilon}^{i}(u)_{+}\right)^{\frac{1}{2}}-\varepsilon^{\frac{N}{2}}\left(c_{i}+\sigma_{i}\right)^{\frac{1}{2}}\right\}_{+}^{2}
$$

The constant $M$ will be chosen later. Finally the penalized functional $E_{\varepsilon}: H \rightarrow \mathbb{R}$ is defined as

$$
F_{c}(u)=J_{c}(u)+P_{\varepsilon}(u)
$$

The functionals $J_{\varepsilon}$ and $P_{\varepsilon}$ are of class $C^{1}$ and so is $E_{\varepsilon}$. We show next that $E_{\varepsilon}$ has good compactness properties, that is $E_{\varepsilon}$ satisfies the Palais Smale condition.

LEMMA 1.1. - Let $\left\{u_{n}\right\}$ be a sequence in $H$ such that $E_{\varepsilon}\left(u_{n}\right)$ is bounded and $E_{\varepsilon}{ }^{\prime}\left(u_{n}\right) \rightarrow 0$. Then $u_{n}$ has a convergent subsequence. 
Proof. - We first prove that the sequence $\left\{u_{n}\right\}$ is bounded in $H$. Using property $(\mathrm{g} 3)$ we easily see that

$$
J_{\varepsilon}\left(u_{n}\right)-\frac{1}{\theta} J_{\varepsilon}^{\prime}\left(u_{n}\right) u_{n} \geq \frac{\theta-2}{2 \theta}\left(\int_{\Omega} \varepsilon^{2}\left|\nabla u_{n}\right|^{2}+V(x) u_{n}^{2}-\int_{\Omega \backslash \Lambda} g\left(x, u_{n}\right) u_{n}\right),
$$

from where, using (g3) again, we obtain

$$
J_{\varepsilon}\left(u_{n}\right)-\frac{1}{\theta} J_{\varepsilon}^{\prime}\left(u_{n}\right) u_{n} \geq C \int_{\Omega} \varepsilon^{2}\left|\nabla u_{n}\right|^{2}+V(x) u_{n}^{2} .
$$

In a similar fashion we find

$$
J_{\varepsilon}^{i}\left(u_{n}\right)-\frac{1}{\theta} J_{\varepsilon}^{i^{\prime}}\left(u_{n}\right) u_{n} \geq C \int_{\tilde{\Lambda}_{i}} \varepsilon^{2}\left|\nabla u_{n}\right|^{2}+V(x) u_{n}^{2} .
$$

We observe that (1.13) still holds if we replace $\theta$ by any number $\hat{\theta} \in(2, \theta)$. Then, for the penalization functional we have

$$
\begin{aligned}
P_{\varepsilon}\left(u_{n}\right)-\frac{1}{\hat{\theta}} P_{\varepsilon}^{\prime}\left(u_{n}\right) u_{n} \geq & -M \varepsilon^{\frac{N}{2}}\left(c_{i}+\sigma_{i}\right)^{\frac{1}{2}} \sum_{i=1}^{K} \\
& \times\left\{\left(J_{\varepsilon}^{i}\left(u_{n}\right)_{+}\right)^{\frac{1}{2}}-\varepsilon^{\frac{N}{2}}\left(c_{i}+\sigma_{i}\right)^{\frac{1}{2}}\right\}_{+} \\
\geq & -C \varepsilon^{\frac{N}{2}} P_{\varepsilon}\left(u_{n}\right)^{\frac{1}{2}}
\end{aligned}
$$

From where there exists a constant $C$ so that

$$
P_{\varepsilon}\left(u_{n}\right)-\frac{1}{\theta} P_{\varepsilon}^{\prime}\left(u_{n}\right) u_{n} \geq-C .
$$

We observe that the constant $C$ is a multiple of $\varepsilon^{N}$.

Thus, it follows from (1.12), (1.15) and the assumptions on $\left\{u_{n}\right\}$ that this sequence is bounded as desired.

Let us choose a subsequence, still denoted by $\left\{u_{n}\right\}$, weakly convergent to $u$ in $H$. This convergence is actually strong. Indeed, it suffices to show that, given $\gamma>0$, there is an $R>0$ such that

$$
\limsup _{n \rightarrow \infty} \int_{\Omega \backslash B_{R}}\left\{\varepsilon^{2}\left|\nabla u_{n}\right|^{2}+V(x) u_{n}^{2}\right\}<\gamma,
$$

where $B_{R}$ denotes the ball with center 0 and radius $R$. We may assume that $R$ is chosen so that $\Lambda \subset B_{R / 2}$. 
Let $\eta_{R}$ be a cut-off function so that $\eta_{R}=0$ on $B_{R / 2}, \eta_{R}=1$ on $\Omega \backslash B_{R}, 0 \leq \eta_{R} \leq 1$ and $\left|\nabla \eta_{R}\right| \leq c / R$. Since $\left\{u_{n}\right\}$ is a P.S. sequence, we have that

$$
<E_{c}^{\prime}\left(u_{n}\right) \cdot \eta_{R} u_{n}>=o_{n}(1)
$$

where $o_{n}(1) \rightarrow 0$ as $n \rightarrow \infty$. Then, as $\left\{u_{n}\right\}$ is bounded

$$
\begin{aligned}
& \int_{\Omega}\left\{\varepsilon^{2}\left|\nabla u_{n}\right|^{2}+V(x) u_{n}^{2}\right\} \eta_{R}+\int_{\Omega} u_{n} \nabla u_{n} \cdot \nabla \eta_{R}= \\
& =\int_{\Omega} g\left(x, u_{n}\right) u_{n} \eta_{R}+o_{n}(1) \leq \frac{1}{2} \int_{\Omega} V(x) u_{n}^{2} \eta_{R}+o_{n}(1) .
\end{aligned}
$$

We conclude that

$$
\int_{\Omega \backslash B_{R}} \varepsilon^{2}\left|\nabla u_{n}\right|^{2}+V(x) u_{n}^{2} \leq \frac{C}{R}\left\|u_{n}\right\|_{L^{2}(\Omega)}\left\|\nabla u_{n}\right\|_{L_{2}(\Omega)}+o_{n}(1) .
$$

from where (1.16) follows.

The previous lemma makes possible to use Critical Point Theory to find critical points of the functional $E_{\varepsilon}$. We will formulate an appropriate minimax problem for $E_{c}$.

We start defining a class of functions $\Gamma$ over which we minimax. A continuous function $\gamma:[0,1]^{K} \rightarrow H$ is in $\Gamma$ if there are continuous functions $g_{i}:[0,1] \rightarrow H, i=1, \ldots, K$ such that

(i) $\operatorname{supp}\left\{g_{i}(\tau)\right\} \subset \Lambda_{i}$ for all $\tau \in[0.1]$,

(ii) $\gamma\left(\tau_{1}, \ldots, \tau_{K}\right)=\sum_{i=1}^{K} g_{i}\left(\tau_{i}\right)$ for all $\left(\tau_{1}, \ldots, \tau_{K^{-}}\right) \in \partial[0.1]^{K}$,

(iii) $g_{i}(0)=0$ and $J_{c}\left(g_{i}(1)\right)<0$

(iv) $E_{\varepsilon}(\gamma(t)) \leq \varepsilon^{N}\left(\sum_{i=1}^{K} c_{i}-\sigma\right)$ for all $t \in \partial[0,1]^{K}$, where $0<\sigma \leq \frac{1}{2} \operatorname{Min}\left\{c_{i} \mid i=1, \ldots, K\right\}$ is a fixed number.

We can define the minimax value associated to the class $\Gamma$ as follows

$$
C_{\varepsilon}=\inf _{\gamma \in \Gamma} \sup _{t \in[0, T]^{\kappa}} E_{\varepsilon}(\gamma(t))
$$

In the following lemma we provide the key estimates on the minimax value $C_{\varepsilon}$. In particular we show that $\Gamma$ is nor empty.

LEMMA 1.2.

$$
C_{\varepsilon}=\varepsilon^{N}\left(\sum_{i=1}^{K} c_{i}+o(1)\right)
$$


Here, and in what follows, we denote by o(1) a quantity approaching zero as $\varepsilon \rightarrow 0$.

The proof of this lemma will require the study of an auxiliary Neumann problem. We may consider the the functional $J_{\varepsilon}^{i}$ on $I^{1}\left(\tilde{\Lambda}_{i}\right)$. Let $d_{\varepsilon}^{i}$ be the mountain pass value of $J_{i}^{\varepsilon}$, namely

$$
d_{\varepsilon}^{i}=\inf _{\gamma_{i} \in \Gamma_{i}} \sup _{\tau \in[0,1]} J_{\varepsilon}^{i}\left(\gamma_{i}(\tau)\right)
$$

where $\Gamma_{i}$ is the class of all continuous curves $\gamma_{i}:[0,1] \rightarrow H^{1}\left(\tilde{\Lambda}_{i}\right)$ such that $\gamma_{i}(0)=0$ and $J_{\varepsilon}^{i}\left(\gamma_{i}(1)\right)<0$. Then we have

LEMMA 1.3. - The mountain pass critical value $d_{\varepsilon}^{i}$ of the Neumann problem satisfies

$$
d_{\varepsilon}^{i}=\left(c_{i}+o(1)\right) \varepsilon^{N}
$$

For the sake of continuity in the arguments, we postpone the proof of this lemma to the Appendix.

Proof of Lemma 1.2. - Since $c_{i}$ is the mountain pass value for the limiting functional $I^{b_{i}}$, given any $\delta>0$ there exists a path $\gamma_{i}:[0,1] \rightarrow H^{1}\left(\mathbf{R}^{N}\right)$ such that $\gamma_{i}(0)=0, I^{b_{i}}\left(\gamma_{i}(1)\right)<0$ and

$$
c_{i} \leq \max _{\tau \in[0,1]} I^{b_{i}}\left(\gamma_{i}(\tau)\right) \leq c_{i}+\frac{\delta}{2 K} .
$$

We assume from now on that $\delta<\min \left\{\sigma, K \sigma_{i}\right\}$. Next, given $\varepsilon>0$, we define the path $\hat{\gamma}_{i}:[0,1] \rightarrow H$ as

$$
\hat{\gamma}_{i}(\tau)(x)=\eta_{i}(x) \gamma_{i}(\tau)\left(\frac{x-x_{i}}{\varepsilon}\right), \quad x \in \Omega
$$

Here $x_{i} \in \Lambda_{i}$ satisfies $b_{i}=V\left(x_{i}\right)$, and $\eta_{i}$ is a $C^{\infty}$ cut off function with compact support in $\Lambda_{i}$, taking the value 1 except for a small neighborhood of $\partial \Lambda_{i}$. It is not hard to check that

$$
J_{\varepsilon}\left(\hat{\gamma}_{i}(\tau)\right)=\varepsilon^{N}\left(I^{b_{i}}\left(\gamma_{i}(\tau)\right)+o(1)\right), \quad \text { for all } \quad \tau \in[0,1]
$$

with $o(1)$ independent of $\tau \in[0,1]$.

Now we define the continuous function $\gamma_{0}:[0,1]^{K} \rightarrow H$ as

$$
\gamma_{0}\left(\tau_{1}, \ldots, \tau_{K}\right)=\sum_{i=1}^{K} \hat{\gamma}_{i}\left(\tau_{i}\right), \quad \text { for all } \quad\left(\tau_{1}, \ldots, \tau_{K^{*}}\right) \in[0,1]^{K^{*}}
$$


The function $\gamma_{0}$ belongs to $\Gamma$. In fact, properties (i)-(ii) are trivially true, and (iii)-(iv) are satisfied in view of (1.19) and (1.20), when $\varepsilon$ is small enough. Thus, the minimax value $C_{i}$ is well defined as a real number, for all $\varepsilon>0$ small enough.

We observe that, by the choice of $\delta$ and from (1.19) and (1.20), the penalization term in $E_{\varepsilon}$ vanishes on $\gamma_{0}(t)$ for all $t \in[0.1]^{K^{\circ}}$.

We also obtain from (1.19) and (1.20), that for small $\varepsilon$

$$
E_{\varepsilon}\left(\gamma_{0}(t)\right) \leq \varepsilon^{N}\left(\sum_{i=1}^{K} c_{i}+\delta\right) . \quad \text { for all } t \in[0,1]^{K} .
$$

so that

$$
\limsup _{\varepsilon \rightarrow 0} \frac{C_{\varepsilon}}{\varepsilon^{N}} \leq \sum_{i=1}^{K^{-}} c_{i}+\delta
$$

As $\delta$ is arbitrary, we obtain the upper estimate $C_{c} \leq \varepsilon^{N}\left(\sum_{i=1}^{K} c_{i}+o(1)\right)$.

We prove the lower estimate next. First we observe that given any $\gamma \in I$ and any curve $c(s)$ joining $\{0\} \times[0, T]^{K-1}$ with $\{T\} \times[0, T]^{K-1}$, the path

$$
\gamma_{1}=\gamma \circ h_{i_{1}} \text { is in } \Gamma_{1} \text {. }
$$

and then it follows from Lemma 1.3 that

$$
\sup _{\tau \in[0.1]} J_{\varepsilon}^{1}\left(\gamma_{1}(\tau)\right) \geq \varepsilon^{N}\left(c_{1}+o(1)\right)
$$

We have an inequality of this form for every $i=1, \ldots, K$. Thus we can repeat the argument given by Coti-Zelati and Rabinowitz in [2] in the proof of Proposition 3.4 to obtain, for every $\gamma \in \Gamma$, the existence of a point $\bar{t} \in[0, T]^{K}$ such that

$$
J_{\varepsilon}^{i}(\gamma(\bar{t})) \geq \varepsilon^{N}\left(c_{i}+o(1)\right) \quad \text { for all } \quad i=1 \ldots K .
$$

From here, and from the form of $J_{\varepsilon}$ outside the $\hat{\Lambda}_{i}$ 's, we have

$$
\sup _{t \in[0, T]^{K}} J_{\varepsilon}(\gamma(t)) \geq \sup _{t \in[0, T]^{K}} \sum_{i=1}^{K} J_{\varepsilon}^{i}(\gamma(t)) \geq \sum_{i=1}^{K} J_{\varepsilon}^{i}(\gamma(\bar{t})) \geq \varepsilon^{N} \sum_{i=1}^{K}\left(c_{i}+o(1)\right)
$$

finishing the proof. 
The functional $E_{\varepsilon}$ satisfies the Palais Smale condition, the class $\Gamma$ is not empty, and estimate (1.17) holds, then there exits a critical point $u_{\varepsilon} \in H$ of $E_{\varepsilon}$ such that $E_{\varepsilon}\left(u_{\varepsilon}\right)=C_{\bar{c}}$.

We define the local weight

$$
\mathrm{w}_{\varepsilon}^{i}=M\left\{\left(J_{\varepsilon}^{i}\left(u_{\varepsilon}\right)_{+}\right)^{\frac{1}{2}}-\varepsilon^{\frac{N}{2}}\left(c_{i}+\sigma_{i}\right)^{\frac{1}{2}}\right\}_{+}\left(J_{\varepsilon}^{i}\left(u_{\varepsilon}\right)\right)^{-\frac{1}{2}} .
$$

and then the function

$$
\mathrm{w}_{\varepsilon}=\sum_{i=1}^{K} \mathrm{w}_{\varepsilon}^{i} \chi_{\hat{\Lambda}_{i}}
$$

The critical point $u_{\varepsilon}$ is a weak solution of the equation

$$
\varepsilon^{2} \operatorname{div}\left(\left(1+\mathrm{w}_{\varepsilon}\right) \nabla u\right)-\left(1+\mathrm{w}_{\varepsilon}\right) V(x) u+\left(1+\mathrm{w}_{\varepsilon}\right) g(x, u)=0 \quad \text { in } \quad \Omega,(1.22)
$$

so that $u_{\varepsilon}$ satisfies

$$
\varepsilon^{2} \Delta u-V(x) u+g(x, u)=0 \text { in } \mathcal{O} .
$$

for every set $\mathcal{O} \subset \Omega$ not intersecting $\partial\left(\cup_{i=1}^{K} \tilde{\Lambda}_{i}\right)$.

We define the sets $\Omega_{\varepsilon}=\left\{y \in \mathbb{R}^{N} / \varepsilon y \in \Omega\right\}, \Lambda_{i}^{\varepsilon}=\left\{y \in \mathbb{R}^{N} / \varepsilon y \in \Lambda_{i}\right\}$ and $\tilde{\Lambda}_{i}=\left\{y \in \mathbb{R}^{N} / \varepsilon y \in \tilde{\Lambda}_{i}\right\}$. We rescale the function $u_{\varepsilon}$ as $v_{\varepsilon}(y)=u_{\varepsilon}(\varepsilon y)$ for $y \in \Omega_{\varepsilon}$. This function $v_{\varepsilon}$ belongs to $H_{0}^{1}\left(\Omega_{\varepsilon}\right)$ and then to $H^{1}\left(\mathbb{R}^{N}\right)$, and it satisfies in a weak sense the equation

$$
\begin{aligned}
\operatorname{div}\left(\left(1+\mathrm{w}_{\varepsilon}(\varepsilon y)\right) \nabla u\right) & -\left(1+\mathrm{w}_{\varepsilon}(\varepsilon y)\right) V(\varepsilon y) u \\
& +\left(1+\mathrm{w}_{\varepsilon}(\varepsilon y)\right) g(\varepsilon y \cdot u)=0 \text { in } \Omega_{\varepsilon}
\end{aligned}
$$

and over sets $\mathcal{O}$, subsets of $\Omega_{\varepsilon}$ not intersecting $\partial\left(\cup_{i=1}^{K} \tilde{\Lambda}_{i}^{\xi}\right)$, the function $v_{\varepsilon}$ satisfies

$$
\Delta u-V(\varepsilon y) u+g(\varepsilon y, u)=0 \quad \text { in } \mathcal{O} .
$$

Finally, proceeding as in the first part of the proof of Lemma 1.1, we obtain from the estimates on $C_{\varepsilon}$ given in Lemma 1.2 that

$$
\int_{\Omega} \varepsilon^{2}\left|\nabla u_{\varepsilon}\right|^{2}+u_{\varepsilon}^{2} \leq C \varepsilon^{N}
$$

and then for the function $v_{\varepsilon}$ we have the uniform $H^{1}$-estimate

$$
\int_{\Omega_{\varepsilon}}\left|\nabla v_{\varepsilon}\right|^{2}+v_{\varepsilon}^{2} \leq C .
$$

Vot. $15, \mathrm{n}^{\circ} 2-1998$. 


\section{PROOF OF THEOREM 0.1}

In this section we will carry out the proof of Theorem 0.1 . Using the estimates obtained in the previous section, we will show that if $M$ in the definition of $E_{\varepsilon}$ is chosen a priori sufficiently large, then $u_{\varepsilon}$ will be a critical point of the original functional $I_{\varepsilon}$ whenever $\varepsilon$ is sufficiently small. Toward this end, the following lemma constitutes a crucial step. It tells us in particular that if $M$ was large then the penalization term $P_{\varepsilon}\left(u_{\varepsilon}\right)$ becomes zero for all sufficiently small $\varepsilon$.

Lemma 2.1. - If in the definition of $E_{\varepsilon}$ in (1.10) and (1.II), M>0 was chosen sufficiently large, then

$$
\limsup _{\varepsilon \rightarrow 0} J_{\varepsilon}^{i}\left(u_{\varepsilon}\right) \varepsilon^{-N} \leq c_{i}+\sigma_{i}, \text { for all } i=1, \ldots, K .
$$

For the proof of this result some preliminaries are required. It is useful to work with the rescaling of $u_{\varepsilon}$ given by $v_{\varepsilon}$, as defined at the end of the last section. Given $R>0$, we denote by $N_{R}\left(\Lambda^{\varepsilon}\right)$ the set $\left\{y \mid \operatorname{dist}\left(y, \Lambda^{\varepsilon}\right)<R\right\}$, a similar definition has the set $N_{R}\left(\Lambda_{i}^{\bar{j}}\right)$. The next lemma states that $v_{\varepsilon}$ is small in $H^{1}$-norm away from the set $\Lambda^{\varepsilon}=\cup_{i=1}^{K} \Lambda_{i}^{\varepsilon}$.

LEMMA 2.2. - There exists a $C>0$ such that, given $R>0$, one has

$$
\int_{\Omega_{\varepsilon} \backslash N_{R}\left(\Lambda^{*}\right)}\left|\nabla v_{\varepsilon}\right|^{2}+v_{\varepsilon}^{2} \leq \frac{C}{R}
$$

for all $\varepsilon$ sufficiently small.

Proof. - Given $R>0, \varepsilon>0$, we may choose smooth cut-off functions $0 \leq \psi_{i, R}^{\ominus} \leq 1$ such that

$$
\psi_{i, R}^{\varepsilon}(y)= \begin{cases}1 & \text { if } \operatorname{dist}\left(y, \Lambda_{i}^{\varepsilon}\right)<R / 2, \\ 0 & \text { if } \operatorname{dist}\left(y, \Lambda_{i}^{\varepsilon}\right)>R\end{cases}
$$

and $\left|\nabla \psi_{i, R}^{\varepsilon}\right| \leq A / R$. Then set $\eta_{R}^{\varepsilon}=1-\sum_{i} \psi_{i, R}^{\varepsilon}$. Using the test function $\eta_{R}^{\varepsilon} v_{\varepsilon}$ in (1.24), equation satisfied weakly by $v_{\varepsilon}$, one gets

$$
\begin{aligned}
& \int_{\Omega_{\varepsilon} \backslash \Lambda^{\varepsilon}}\left(1+\mathrm{w}_{\varepsilon}(\varepsilon y)\right) \eta_{R}^{\varepsilon}\left(\left|\nabla v_{\varepsilon}\right|^{2}+\left\{V(\varepsilon y)-\frac{\tilde{f}\left(v_{\varepsilon}\right)}{v_{\varepsilon}}\right\} v_{\varepsilon}^{2}\right)= \\
& -\int_{\Omega^{\varepsilon} \backslash \Lambda^{\varepsilon}}\left(1+\mathrm{w}_{\varepsilon}(\varepsilon y)\right) \nabla \eta_{R}^{\varepsilon} \nabla v_{\varepsilon} v_{\varepsilon},
\end{aligned}
$$

where $\mathrm{w}_{\varepsilon}$ is given by $(1.21)$. Observe that $\mathrm{w}_{\varepsilon}$ is uniformly bounded, by a bound possibly depending on $M$. Using this, the choice of $\eta_{R}^{\varepsilon}$, the fact that 
$v_{\varepsilon}$ is uniformly bounded in $H^{1}\left(\mathbb{R}^{N}\right)$ and the definition of $\tilde{f}$, the desired estimate (2.2) follows immediately from (2.4).

A second preliminary result we will need is given in the following lemma inspired by the work in [4].

Lemma 2.3. - Let $v \in H^{1}\left(\mathbb{R}^{N}\right) \cap C\left(\mathbb{R}^{N}\right)$ be a solution of the equation

$$
\Delta v-b v+\chi_{\left\{x_{1}<0\right\}} f(v)+\chi_{\left\{x_{1}>0\right\}} \tilde{f}(v)=0 \text {. }
$$

where $b>0$. Then $v \leq a$ for $x_{1}>0$, so that $v$ actually solves

$$
\Delta v-b v+f(v)=0
$$

Proof. - We begin by showing that $v \leq a$ on $\left\{x_{1}=0\right\}$. Standard regularity arguments yield that $v$ is in $C^{1}\left(\mathbb{R}^{N}\right) \cap H^{2}\left(\mathbb{R}^{N}\right)$. Moreover, $v(x), \nabla v(x) \rightarrow 0$ as $|x| \rightarrow \infty$.

Using $\frac{\partial_{v}}{\partial x_{1}}$ as a test function in equation (2.5), we obtain

$$
\begin{aligned}
& \int_{\mathbb{R}^{v} \cdots 1} d x^{\prime} \int_{-\infty}^{\infty} \frac{\partial}{\partial x_{1}}\left(|\nabla v|^{2}+b v^{2}\right) d x_{1} \\
& \quad+\int_{\mathbb{R}^{N-1}}\left(F\left(v\left(0, x^{\prime}\right)\right)-\tilde{F}\left(v\left(0, x^{\prime}\right)\right)\right) d x^{\prime}=0 .
\end{aligned}
$$

But the first summand in the above quantity is zero, while $F(s) \geq \tilde{F}(s)$, with equality only if $s \leq a$. Thus, $v\left(0, x^{\prime}\right) \leq a$. Finally, to prove that $v\left(x_{1}, x^{\prime}\right) \leq a$ for $x_{1} \geq 0$, we just consider the test function $\phi=(v-a)_{+} \chi_{\left\{x_{1}>0\right\}} \in H^{1}\left(\mathbb{R}^{N}\right)$ in equation (2.5), and the conclusion $\phi \equiv 0$ readily follows.

Proof of Lemma 2.1. - We will base the proof in an indirect argument. Thus, assume that (2.1) does not hold, namely that for some sequence $\varepsilon_{j} \rightarrow 0$ we have

$$
\lim _{j \rightarrow \infty} J_{\tilde{\varepsilon}_{i}}^{i}\left(u_{\varepsilon_{j}}\right) \varepsilon_{j}{ }^{-N}>c_{i}+\sigma_{i}
$$

We will see that (2.7) is impossible provided that $M$ was chosen sufficiently large. In fact this is a consequence of the following claim, main step in the proof: if (2.7) holds, then

$$
\liminf _{j \rightarrow \infty} J_{\varepsilon_{j}}^{i}\left(u_{\varepsilon_{j}}\right) \varepsilon_{j}{ }^{-N} \geq 2 c_{i} .
$$


We will show (2.8). We start proving that the sequence $u_{\varepsilon_{j}}$ concentrates somewhere near $\Lambda_{i}^{\varepsilon_{j}}$, more precisely, we show that there exist numbers $S>0, \rho>0$ such that

$$
\sup _{y \in \Lambda_{j}^{\varepsilon_{j}}} \int_{B \hookrightarrow(y)} v_{\varepsilon_{j}}^{2} \geq \rho \text { for all } j \geq j_{0}
$$

To see this, we first observe that from $(2.7)$, there is a $\lambda>0$ such that

$$
\int_{\tilde{\Lambda}_{i}}\left|\nabla v_{\varepsilon_{j} j}\right|^{2}+v_{\varepsilon_{j}}^{2} \geq \lambda
$$

then, Lemma 2.2 imply that for all $R>0$ large enough

$$
\int_{\Lambda_{k}\left(\Lambda_{i}^{j}\right)}\left|\nabla v_{\varepsilon_{j}}\right|^{2}+v_{\varepsilon_{j}}^{2} \geq \frac{\lambda}{2}
$$

Now assume that (2.9) is false. Then we may assume that for all $S>0$ we have

$$
\sup _{y \in \Lambda_{i}} \int_{B_{s}(y)} v_{\varepsilon_{j}}^{2} \rightarrow 0
$$

Let us set $v_{j}^{R}=\psi_{R}^{j} v_{\varepsilon_{j}}$, where $\psi_{R}^{j}=\psi_{i, 2 R}^{\varepsilon_{j}}$ is given by (2.3). Then clearly

$$
\sup _{y \in \mathbb{R}^{N}} \int_{B s(y)}\left(v_{j}^{R}\right)^{2} \rightarrow 0
$$

for all $S>0$. Moreover, $\left\{v_{j}^{R}\right\}$ is a bounded sequence in $H^{1}\left(\mathbb{R}^{N}\right)$. Then applying the concentration compactness principle (see Lemma I.1 in [7] or Lemma 2.18 in [2]), we obtain that

$$
\int_{\mathbb{R}^{N}}\left(v_{j}^{R}\right)^{q} \rightarrow 0 \text { for all } 2<q<2 N /\left(\begin{array}{ll}
N & 2
\end{array}\right),
$$

for each $R>0$, then, in particular

$$
\int_{N_{R}\left(\Lambda_{i}^{j}\right)} v_{\varepsilon_{i}}^{s+1} \rightarrow 0
$$

where $s$ is as in (f2). Using $v_{j}^{R}$ as a test function in (1.25), the equation satisfied by $v_{\varepsilon_{j}}$, we get

$$
\begin{aligned}
& \int_{\tilde{\Lambda}_{i}^{\varepsilon_{j}}}\left\{\left|\nabla v_{\varepsilon_{j}}\right|^{2}+V\left(\varepsilon_{j} y\right) v_{\varepsilon_{j}}^{2}\right\} \psi_{R}^{j} \\
& \quad=-\int_{\tilde{\Lambda}_{i}^{\varepsilon_{j}}} v_{\varepsilon_{j}} \nabla \psi_{R}^{j} \nabla v_{\varepsilon_{j}}+\int_{\tilde{\Lambda}_{i}^{\varepsilon_{j}}} g\left(\varepsilon_{j} y, v_{\varepsilon_{j}}\right) v_{\varepsilon_{j}} \psi_{R}^{j} \leq \frac{C}{R}+\int_{N_{2 R}\left(\Lambda_{i}^{j}\right)} v_{\varepsilon_{j}}^{s+1} .
\end{aligned}
$$


Hence, choosing $R$ and $j$ large enough, we obtain from the above estimate, an immediate contradiction with (2.10). This shows the validity of (2.9). Thus, we may assume there is a sequence $y_{j} \in \Lambda_{i}^{\varepsilon_{j}}$ with

$$
\int_{B_{S}\left(y_{j}\right)} v_{\varepsilon_{j}}^{2} \geq \rho>0 \text { for all } j \geq j_{0}
$$

Let us now write $v_{j}(y)=v_{\varepsilon_{j}}\left(y_{j}+y\right)$. Since $v_{j}$ is a bounded sequence in $H^{1}\left(\mathbb{R}^{N}\right)$, we may assume it converges weakly to a $v \in H^{1}\left(\mathbb{R}^{N}\right)$. Assume first that

$$
\operatorname{dist}\left(y_{j}, \partial \Lambda_{i}^{\varepsilon_{j}}\right) \rightarrow \infty
$$

Set $x_{j}=\varepsilon_{j} y_{j} \in \Lambda_{i}$ and assume that $x_{j} \rightarrow \bar{x} \in \bar{\Lambda}_{i}$. Since $v_{j}$ satisfies in $\left\{-y_{j}\right\}+\Lambda_{i}^{\varepsilon_{j}}$ the equation

$$
\Delta v_{j}-V\left(x_{j}+\varepsilon_{j} y\right) v_{j}+f\left(v_{j}\right)=0
$$

it follows that $v$ satisfies in $\mathbb{R}^{N}$,

$$
\Delta v-b v+f(v)=0
$$

where $b=V(\bar{x})$. Moreover, $v \not \equiv 0$, thanks to (2.13). On the other hand, if $\operatorname{dist}\left(y_{j}, \partial \Lambda_{i}^{\varepsilon_{3}}\right) \leq C<\infty$, we will have that $v$ satisfies an equation of the form (2.5), so that Lemma 2.3 implies that $v$ satisfies (2.15). In both cases $v$ is the unique critical point of the functional $I^{b}$ defined in (1.7), with $b \leq \sup \left\{V(x) \mid x \in \Lambda_{i}\right\} \leq b_{i}+\delta$. Then we have

$$
c_{i} \leq I^{b}(v) \leq c_{i}+\sigma_{i}
$$

On the other hand, elliptic regularity implies, in particular, that $v_{\varepsilon_{j}}$ converges strongly in the $H^{1}$-sense over compacts. Passing to a further subsequence if necessary, we may find a sequence of positive numbers $R_{j} \rightarrow+\infty$ such that

$$
\lim _{j \rightarrow \infty} \int_{B_{R_{j}}\left(y_{j}\right)}\left(\frac{1}{2}\left\{\left|\nabla v_{c_{j}}\right|^{2}+V\left(\varepsilon_{j} y\right) v_{\varepsilon_{j}}^{2}\right\}-G\left(\varepsilon_{j} y, v_{c_{j}}\right)\right) d y=I^{b}(v) \leq c_{i}+\sigma_{i}
$$

Thus, combining (2.7) and (2.16) we find that there cxists $\eta>0$ such that for all large $j$

$$
0<\eta<\int_{\tilde{\Lambda}_{i}^{\varepsilon_{j}} \backslash B_{R_{j}}\left(\boldsymbol{y}_{j}\right)}\left|\nabla v_{\varepsilon_{j}}\right|^{2}+v_{\tilde{\varepsilon}_{j}}^{2}
$$


Using (2.17) and applying a concentration-compactness argument, similar to that we used above, to the sequence obtained after multiplying $v_{c}$, by a suitable cut-off function vanishing on $B_{R_{j}}\left(y_{j}\right)$, we will end up with the existence of an $S>0$ and a sequence $\tilde{y}_{j} \in \Lambda_{i}^{\varepsilon^{\prime}} \backslash B_{R_{j}}\left(y_{j}\right)$ such that

$$
\int_{B_{K}\left(\tilde{y}_{j}\right)} v_{\varepsilon_{i}}^{2} \geq \rho>0 .
$$

Thus, we have again, after passing to a subsequence, the weak convergence of $v_{\varepsilon, j}\left(\cdot+\tilde{y}_{j}\right)$ to a nonzero $\hat{v} \in H^{1}\left(\mathbb{R}^{N}\right)$. Moreover, $\hat{v}$ satisfies the equation

$$
\Delta \hat{v}-b \hat{v}+f(\hat{v})=0 \text {. }
$$

where $\hat{b}=V(\hat{x})$, with $\hat{x} \in \bar{\Lambda}_{i}$. From $(2.18) \hat{v} \not \equiv 0$, hence $I^{b}(\hat{v}) \geq c_{i}$. Our next claim is that

$$
\lim _{j \rightarrow \infty} J_{c_{j}}^{i}\left(u_{r_{j}}\right) \varepsilon_{j}{ }^{-N} \geq I^{b}(v)+I^{i}(\hat{v}) \geq 2 c_{i}
$$

To verify this, we recall that $v_{c}$, satisfies on $\bar{\Lambda}_{i}^{\varepsilon_{j}}$ the equation

$$
\Delta v_{c_{j}}-V\left(\varepsilon_{j} y\right) v_{\varepsilon_{j}}+g\left(\varepsilon_{j} y, v_{c_{j}}\right)=0 .
$$

We use in this equation a test function of the form

$$
\phi=v_{c_{j}}\left\{\psi\left(\left|y-y_{j}\right| / R\right)+\psi\left(\left|y-\tilde{y}_{j}\right| / R\right)\right\}
$$

where $\psi$ is a $C^{\infty}$ function with $\psi(s)=0$ for $s \leq 1$ and $\psi(s)=1$ for $s \geq 2$. Denoting $N_{R}\left(y_{j}, y_{j}\right)=B\left(y_{j}, R\right) \cup B\left(y_{j}, R\right)$, the conclusion we obtain, after a direct estimate is that

$$
\begin{aligned}
& \int_{\tilde{\Lambda}_{i}^{\varepsilon_{j}} \backslash N_{2 R}\left(y_{j}, \tilde{y}_{j}\right)}\left|\nabla v_{\varepsilon_{j}}\right|^{2}+V\left(\varepsilon_{j} y\right) v_{\varepsilon_{j}}^{2} \\
& \geq \int_{\bar{i}_{i},{ }_{j} \backslash N_{k}\left(y_{j}, \dot{y}_{i}\right)} g\left(\varepsilon_{j} y, v_{\varepsilon_{j}}\right) v_{\varepsilon_{j}}+O\left(\frac{1}{R}\right) \\
& \geq 2 \int_{\bar{\Lambda}_{2}, \lambda_{R}\left(y_{j}, \bar{y}_{j}\right)} G\left(\varepsilon_{j} y, v_{\varepsilon_{j}}\right)+O\left(\frac{1}{R}\right) .
\end{aligned}
$$

Then, it follows that

$$
\begin{aligned}
& J_{\varepsilon_{j}}^{i}\left(u_{\varepsilon_{j}}\right) \varepsilon_{j}^{-N} \\
& \quad \geq \int_{N_{R}\left(\boldsymbol{y}_{j}, \tilde{y}_{j}\right)}\left(\frac{1}{2}\left\{\left|\nabla v_{\varepsilon_{j}}\right|^{2}+V\left(\varepsilon_{j} y\right) v_{\varepsilon_{j}}^{2}\right\}-G\left(\varepsilon_{j} y, v_{\varepsilon_{j}}\right)\right) d y+O\left(\frac{1}{R}\right)
\end{aligned}
$$


so that

$$
\begin{aligned}
\lim _{j \rightarrow \infty} J_{\varepsilon_{j}}^{i}\left(u_{\varepsilon_{j}}\right) \varepsilon_{j}{ }^{-N} & \geq \int_{B_{R}(0)}\left(\frac{1}{2}\left\{|\nabla v|^{2}+b v^{2}\right\}-F(v)\right) \\
& +\int_{B_{R}(0)}\left(\frac{1}{2}\left\{|\nabla \hat{v}|^{2}+\hat{b} \hat{v}^{2}\right\}-F(\hat{v})\right)+O\left(\frac{1}{R}\right),
\end{aligned}
$$

from where (2.19) follows since $R$ is arbitrary, and the claim (2.8) is thus checked.

We observe that a similar argument applies to the functional $J_{\varepsilon}$, so we also have

$$
\liminf _{j \rightarrow \infty} J_{\varepsilon_{j}}\left(u_{\varepsilon_{j}}\right) \varepsilon_{j}^{-N} \geq 2 c_{i}
$$

The definition of the total functional $E_{\varepsilon}$ in (1.10) and (1.11), thus yields

$$
\liminf _{j \rightarrow \infty} E_{\varepsilon_{j}}\left(u_{\varepsilon_{j}}\right) \varepsilon_{j}{ }^{-N} \geq 2 c_{i}+M\left\{\left(2 c_{i}\right)^{1 / 2}-\left(c_{i}+\sigma_{i}\right)^{1 / 2}\right\}_{+}^{2} .
$$

But, using the upper estimate in the critical value $C_{\varepsilon_{j}}=E_{\varepsilon_{j}}\left(u_{\varepsilon_{j}}\right)$ provided by Lemma 1.2, and the inequality above, we obtain

$$
M\left\{\left(2 c_{i}\right)^{1 / 2}-\left(c_{i}+\sigma_{i}\right)^{1 / 2}\right\}_{+}^{2} \leq \sum_{i=1}^{K} c_{i}
$$

Therefore, if $M$ was such that

$$
M>\frac{\sum_{i=1}^{K} c_{i} .}{\min \left\{\left(\left(2 c_{i}\right)^{1 / 2}-\left(c_{i}+\sigma_{i}\right)^{1 / 2}\right)^{2} \mid i=1, \ldots, K\right\}},
$$

we obtain that our original assumption (2.7) was impossible. This concludes the proof of Lemma 2.1 .

We assume in the rest of this section that $M$ was chosen so large that Lemma 2.1 holds true. Our next lemma is

LEMMA 2.4

$$
\lim _{\varepsilon \rightarrow 0} J_{\varepsilon}^{i}\left(u_{\varepsilon}\right) \varepsilon^{-N}=c_{i} \text { for all } i=1, \ldots, K .
$$

Proof. - We begin by showing

$$
\liminf _{\varepsilon \rightarrow 0} J_{\varepsilon}^{i}\left(u_{\varepsilon}\right) \varepsilon^{-N} \geq c_{i} \quad \text { for all } \quad i=1, \ldots, K \text {. }
$$


Notice that it suffices to show that

$$
\liminf _{\varepsilon \rightarrow 0} J_{c}^{i}\left(u_{\varepsilon}\right) \varepsilon^{-N}>0 \text { for all } i=1 \ldots, k \text {. }
$$

In fact, concentration arguments similar to those employed in the proof of Lemma 2.1 give that then

$$
\liminf _{\varepsilon \rightarrow 0} J_{\varepsilon}^{i}\left(u_{\varepsilon}\right) \varepsilon^{-N} \geq I^{b}(v) \geq c_{i},
$$

where $b=V(\bar{x})$, with $\bar{x} \in \bar{\Lambda}_{i}$.

Then assume that (2.23) does not hold for some $i$, say $i=1$. Then, along a sequence $\varepsilon_{j} \rightarrow 0$ we get

$$
\lim _{j \rightarrow \infty} J_{\varepsilon_{i}}^{1}\left(u_{\varepsilon_{j}}\right) \varepsilon_{j}^{-N^{+}}=0
$$

But Lemma 2.1 implies that, for all $i=1 \ldots, K$

$$
\limsup _{i \rightarrow \infty} \cdot J_{\varepsilon_{j}}^{i}\left(u_{c_{i j}}\right) \varepsilon_{j}^{-N} \leq c_{i}+\sigma_{i}
$$

The definition of $E_{\varepsilon}$, together with (2.25), (2.26), and Lemma 2.2 then yields

$$
\sum_{i=1}^{K} c_{i}=\limsup _{i \rightarrow \infty} J_{\bar{\varepsilon}_{j}}\left(u_{\bar{c}_{j}}\right) \varepsilon_{j}{ }^{-N} \leq \sum_{i=2}^{K} c_{i}+\sigma_{i} .
$$

that is certainly impossible by the choice of the $\sigma_{\text {; }}$ made in (1.8). This concludes the proof of (2.22).

Next we show that (2.21) holds. Assume that for $i=1$ we have along a sequence that

$$
c_{1}+\lambda=\lim _{j \rightarrow \infty} J_{\varepsilon_{j}}^{1}\left(u_{\varepsilon_{j}}\right) \varepsilon_{j}{ }^{-N}>c_{1}
$$

Then we will get, from estimate (2.22) and Lemma 2.1,

$$
\sum_{i=1}^{K} c_{i}=\lim _{j \rightarrow \infty} E_{\varepsilon_{j}}\left(u_{\varepsilon_{j}}\right) \varepsilon_{j}{ }^{-N} \geq\left(c_{1}+\lambda\right)+\sum_{i=2}^{K} c_{i}
$$

which is impossible. This concludes the proof of Lemma 2.4.

Conclusion of the Proof of Theorem 0.1. - To begin with, we show that

$$
\sup _{\partial \Lambda_{i}} u_{\varepsilon} \rightarrow 0 \quad \text { as } \quad \varepsilon \rightarrow 0 \quad \text { for all } i=1, \ldots, K .
$$

In fact, otherwise there would exist a sequence $\varepsilon_{j} \rightarrow 0$ and $x_{j} \in \partial \Lambda_{j}$ with $u_{\varepsilon_{j}}\left(x_{j}\right) \geq \delta>0$. Assuming $x_{j} \rightarrow \bar{x} \in \partial \Lambda_{i}$, and using Lemma 2.3, we 
obtain that the sequence $v_{j}(y)=u_{\varepsilon_{j}}\left(x_{j}+\varepsilon_{j} y\right)$ converges weakly in $H^{1}$ to a nonzero solution $v$ ) of

$$
\Delta v-b v+f(v)=0 .
$$

Since $b=V(\bar{x})>b_{i}$, we have that $I^{b}(v)>c_{i}$. But this imply that

$$
\liminf _{j \rightarrow \infty} J_{\varepsilon_{j}}^{i}\left(u_{\varepsilon_{j}}\right) \varepsilon_{j}^{-N} \geq I^{b}(v)>c_{i}
$$

which is impossible in view of Lemma 2.4. Hence (2.29) holds. Note that (2.29) implies, in particular, that $u_{\varepsilon} \leq a$ on $\partial \Lambda_{i}$ for all $i=1, \ldots, K$, if $\varepsilon$ is sufficiently small.

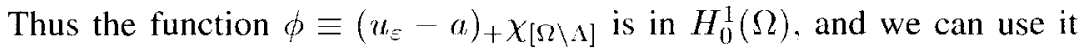
as a test function in (1.22), the equation satisfied by $u_{\xi}$. We immediately conclude that actually $\phi \equiv 0$, in other words, $u \leq a$ on $\Omega \backslash \Lambda$. The conclusion is that $u_{\varepsilon}$ is actually a solution to the original equation $(0.6)$ for all small $\varepsilon$, one of the desired features of $u_{\varepsilon}$.

Finally, the fact that $\lim _{\varepsilon \rightarrow 0} J_{\varepsilon}^{i}\left(u_{\varepsilon}\right) \varepsilon^{-N}=c_{i}>0$ implies that concentration must occur around some $\bar{x} \in \Lambda_{i}$. We must then have $V(\bar{x})=b_{i}$, for otherwise a contradiction similar to (2.28) would arise.

The concentration fact implies the presence of at least one local maximum in each $\Lambda_{i}$, so that $u_{\varepsilon}$ is a $K$-peak solution. The uniqueness of these maxima, as well as the remaining decay assertions of the theorem follow similarly to their analogues shown in [3] for the one-peak case. This concludes the proof of the theorem.

Remark 2.1. - If instead of hypothesis (f4) we assume (f4'), as indicated in the introduction, the proof of Theorem 0.1 can be carried out in a similar way, after choosing $\sigma_{i}$ so small that no critical value of the functional $I^{b}$, other than $c(b)$, exist in $\left(0, c_{i}+3 \sigma_{i}\right)$, for all $b \in\left(b_{i}, b_{i}+\delta\right), i=1, \ldots, K$. We modify the penalization as

$$
P_{\varepsilon}(u)=M \sum_{i=1}^{K}\left\{\left(J_{\varepsilon}^{i}(u)_{+}\right)^{\frac{1}{2}}-\varepsilon^{\frac{\nu}{2}}\left(c_{i}+2 \sigma_{i}\right)^{\frac{1}{2}}\right\}_{+}^{2}
$$

and choose $M$ so that $M \sigma_{i}$ is large enough.

\section{APPENDIX}

We devote the Appendix to the proof of Lemma 1.3. Since many steps in the proof use arguments already given in Section 2, at certain points we don't give all details.

Vol. 15, $n^{\circ} 2-1998$. 
Proof of Lemma 1.3. - An upper estimate of the form

$$
d_{\varepsilon}^{i} \leq\left(c_{;}+o(1)\right) \varepsilon^{N}
$$

follows from the use of a test path constructed as in the beginning of the proof of Lemma 1.2.

On the other hand, it is standard to check that the functional $J_{\varepsilon}^{i}$ satisfies P.S. in $H^{1}\left(\tilde{\Lambda}_{i}\right)$, so that the Mountain Pass Theorem implies that $d_{\varepsilon}^{i}$ is a critical value for it. Let $w_{s}$ be an associated critical point. Then $w_{\varepsilon}$ is a nonzero solution of the equation

$$
\begin{gathered}
\varepsilon^{2} \Delta w-V(x) w+g(x, w)=0 \quad \text { in } \check{\Lambda}_{i} \\
\frac{\partial w}{\partial n}=0 \quad \text { on } \partial \tilde{\Lambda}_{i}
\end{gathered}
$$

We begin by observing that the definition of $g(x, u)$ and the Maximum Principle makes it impossible that $w_{\varepsilon}$ attains a local maximum somewhere in $\operatorname{cl}\left(\tilde{\Lambda}_{i}\right) \backslash \Lambda_{i}$. Thus let $x_{\varepsilon} \in \bar{\Lambda}_{i}$ be a maximum of $w_{\varepsilon}$. Then we have $w_{\varepsilon} \geq \delta>0$, uniformly in $\varepsilon$. We consider the rescaling of $w_{\varepsilon}$ given by $v_{\varepsilon}(y)=w_{\varepsilon}\left(x_{\varepsilon}+\varepsilon y\right)$, and take a sequence $\varepsilon_{j} \rightarrow 0$. Then, for a subsequence of $\varepsilon_{j}$ which we relabel in the same way, we have that $x_{\varepsilon_{j}} \rightarrow \bar{x} \in \bar{\Lambda}_{i}$. Moreover, estimate (3.1) and the fact that $w_{\varepsilon}$ solves equation (3.2) imply that $\left\|w_{\varepsilon}\right\|_{H^{1}} \leq C \varepsilon^{N}$, or equivalently, $\left\|v_{\varepsilon}\right\|_{H^{1}} \leq C$. This fact and elliptic estimates allow us to assume, without loss of generality, that $v_{\varepsilon_{j}} \rightarrow v$ weakly in $H^{1}$ and locally strongly in the $C^{1}$-sense, where $v$ is a nontrivial solution of an equation of the form (2.5), so that Lemma 2.3 is applicable to conclude that $v$ satisfies

$$
\Delta v-V(\bar{x}) v+f(v)=0
$$

Set $b=V(\bar{x})$. Then $b \geq b_{i}$ and, using arguments as in the proof of Lemma 2.1 , we must have

$$
\lim _{j \rightarrow \infty} J_{i}^{\varepsilon_{j}}\left(w_{\varepsilon_{j}}\right) \varepsilon_{j}^{-N} \geq I^{b}(v)
$$

where $I^{b}$ was defined in (1.7).

But $b=V(\bar{x}) \geq b_{i}$, so we have that $I^{b}(v) \geq c_{i}$. Since we have established that every sequence $\varepsilon_{j} \rightarrow 0$ has a subsequence such that (3.3) holds, we then conclude $d_{\varepsilon}^{i} \geq\left(c_{i}+o(1)\right) \varepsilon^{N}$ as desired. This finishes the proof. 


\section{REFERENCES}

[1] C. C. CHEN and C. S. LiN, Uniqueness of the ground state solutions of $\triangle u+f(u)=0$ in $\mathbb{R}^{\wedge}, N \geq 3$. Comm. in P.D.E. 16. Vol. 8-9, 1991, pp. 1549-1572.

[2] V. Con ZtLan and P. Rabinownth. 'Homoclinic type solutions for semilinear elliplic PDE on $\mathbb{R}^{\lambda}$. Comm. Pure and Applied Math, Vol. XLV, 1992, pp. 1217-1269.

[3] M. Del. PINo and P. Felmer, Local mountain passes for semilinear elliptic problems in unbounded domains. Calculus of Variations and PDE. Vol. 4. 1996, pp. 121-137.

[4] M. J. EsTEBAN and P. L. LIONS Existence and non-existence results for semilinear problems in unbounded domains. Proc. Roy. Soc. Edin.. Vol. 93A, 1982, pp. 1-14.

[5] A. Floer and A. Weinstein, Nonspreading Wave Packets for the Cubic Schrödinger Equation with a Bounded Potential, Jommal of Functional analisis, Vol. 69, 1986 , pp. 397-408.

[6] M. K. KWoNG and L. ZHANG, Uniqueness of positive solutions of $\Delta u+f(u)=0$ in an annulus Differential and Integral Equations . Vol. 4, 1991. pp. 583-599.

17] P. L. LioNs, The concentration-compactness principle in the calculus of variations. The locally compact case. Part II Analyse Nonlin.. Vol. 1, 1984, pp. 223-283.

[8] Y. J. OH. Existence of semi-classical bound states of nonlinear Schrödinger equations with potential on the class (V). . Comm. Partial Diff.. Eq. Vol. 13, 1988, pp. 1499-1519.

[9] Y. J. OH. Corrections to Existence of semi-classical bound states of nonlinear Schrödinger equations with potential on the class $(V)_{1, .}$ Comm. Partial Diff. Eq. Vol. 14. 1989. pp. 833834 .

[10] Y. J. ОH, On positive multi-lump bound states nonlinear Schrödinger equations under multiple well potential. Comm. Math. Phys, Vol. 131, 1990. pp. 223-253.

111) P. Rabinowitz, On a class of nonlinear Schrödinger equations, $Z$. angew Math Phys. Vol. 43, 1992, pp. 270-291.

[12] G. SPRADlin, Ph. D. Thesis University of Wisconsin, 1994.

[13] N. Thand)I, Ph. D. Thesis University of Wisconsin. 1995.

[14] X. WANG, On concentration of positive bound states of nonlineal Schrödinger equations. Commr. Math. Phys., Vol. 153, No 2, 1993. pp. 229-244.

(Manuscript received October 23, 1995;

Revised January 10, 1996.)

Vol. $15, n^{\circ} 2-1998$ 